\title{
On Stieltjes-Volterra integral equations
}

\section{S.G. Pandit}

A Stieltjes-Volterra integral equation system

$$
x(t)=f(t)+\int_{t_{0}}^{t} K(t, s, x(s)) d u(s)
$$

is firstly considered. Pointwise estimates and boundedness of its solutions are obtained under various conditions on the function $K$. To do this, the well-known Gronwall-Bellman integral inequality is generalized. For a particular choice of $u$, it is shown that the integral equation reduces to a difference equation. The problem of existence (and nonexistence), uniqueness (and non-uniqueness) of the difference equation is discussed. Gronwall-Bellman inequality is further generalized to $n$ linear terms and is subsequently applied to obtain sufficient conditions in order that a certain stability of the unperturbed Volterra system

$$
x(t)=f(t)+\int_{t_{0}}^{t} a(t, s) x(s) d s
$$

implies the corresponding local stability of the (discontinuously) perturbed system

$$
x(t)=f(t)+\int_{t_{0}}^{t} a(t, s) x(s) d s+\int_{t_{0}}^{t} b(t, s) F(s, x(s)) d u(s) .
$$

Received 11 January 1978. 
1 .

In many problems of physics and engineering (optimal control theory in particular), one can not expect perturbations to be well-behaved and it is therefore important to consider the cases when the perturbations are impulsive [3, 7]. Such systems would be described by differential equations containing measures, which are equivalent to Volterra integral equations with perturbations involving Lebesgue-Stieltjes integrals. The purpose of this paper is to obtain pointwise estimates and boundedness of solutions of Stieltjes-Volterra integral equations and to study a stability property of Volterra integral equations with discontinuous perturbations. The tools used for the purpose are the generalized Gronwall-Bellman inequalities involving Lebesgue-Stieltjes integrals.

Let $J=\left[t_{0}, \infty\right), t_{0} \geq 0$, and $B V\left(J, R^{n}\right)=B V(J)$ denote the space of all functions of bounded variation which are defined on $J$ and taking values in $R^{n}$. The norm of $x=x(t) \in B V(J)$ is defined by $\|x\|=V(x, J)+\left|x\left(t_{0}\right)\right|$ where $V(x, J)$ is the total variation of $x$ on $J$ and $|\cdot|$ is any norm in $R^{n}$. Let $u$ be a scalar function which is right-continuous and of bounded variation on every compact subinterval of $J$. We consider the following Volterra integral equations

(1.1) $x(t)=f(t)+\int_{t_{0}}^{t} K(t, s, x(s)) d u(s)$,

(1.2) $x(t)=f(t)+\int_{t_{0}}^{t} a(t, s) x(s) d s$,

(1.3) $x(t)=f(t)+\int_{t_{0}}^{t} a(t, s) x(s) d s+\int_{t_{0}}^{t} b(t, s) F(s, x(s)) d u(s)$,

where $x, f \in B V(J), K(t, s, \phi): J \times J \times R^{n} \rightarrow R^{n}, F: J \times R^{n} \rightarrow R^{n}$, and $a(t, s), b(t, s)$ are $n \times n$ matrices defined for $t_{0} \leq s \leq t<\infty$. A special case of (1.1) is considered in [2] where the integrals are in the Riemann-Stieltjes sense. (1.2) and (1.3) have been dealt with in [9]. [6, 10] also treat these equations when $a(t, s)=b(t, s)$ and $u$ is 
absolutely continuous on $J$.

In Section 2, we generalize the Gronwall-Bellman integral inequality and apply it to obtain pointwise estimates and boundedness of solutions of (1.1). Section 3 deals with a difference equation arising from (1.1) for a particular choice of $u$. The existence (or non-existence) and uniqueness (or non-uniqueness) of solutions of the difference equation are discussed. Finally, in Section 4, we further generalize the Gronwall-Bellman inequality and study a stability property of (1.3) in the light of (1.2). In the following discussion, it is assumed that (1.1)-(1.3) possess solutions on $J$.

\section{2.}

Let $t_{1}<t_{2}<\ldots$ denote the discontinuities of $u$ on $J$ (note that $u$ is of bounded variation). We assume that the discontinuities are isolated. $u$ may be decomposed as $u=u_{1}+u_{2}$ where $u_{1}$ is an absolutely continuous function of bounded variation on $J$ and $u_{2}$ is a sum of jump functions, the jumps being those of $u$. It follows that $u^{\prime}$ exists (and is equal to $u_{1}^{\prime}$ almost everywhere) on $J$. Let

$\lambda_{k}=u\left(t_{k}\right)-u\left(t_{k}\right)$ denote the jump of $u$ at $t=t_{k}, k=1,2, \ldots$. In the following all functions of one variable are assumed to be defined, real-valued, and measurable on $J$. Such a function $w$ is said to be locally du-integrable on $J$ if, for each $t \in J$, the Lebesgue-Stieltjes integral $\int_{t_{0}}^{t} w(s) d u(s)$ is finite.

THEOREM 2.1. Suppose that

$$
x(t) \leq f(t)+g(t) \int_{t_{0}}^{t} h(s) x(s) d u(s), \quad t \in J,
$$

where

(i) $x, f, g$, and $h$ are non-negative and locally $d u$ integrable on $J$, with $f$ non-decreasing and $g \geq 1$,

(ii) $u$ is such that $u_{1}^{\prime} \geq 0$ on $J$ and 


$$
\lambda_{k} g\left(t_{k}\right) h\left(t_{k}\right)<1, k=1,2, \ldots,
$$

(iii) the series

$$
\sum_{k=1}^{\infty} \lambda_{k} g\left(t_{k}\right) h\left(t_{k}\right)
$$

converges absolutely.

Then

$$
x(t) \leq P^{-1} f(t) g(t) \exp \left(\int_{t_{0}}^{t} g(s) h(s) u_{1}^{\prime}(s) d s\right), \quad t \in J,
$$

where

$$
P=\prod_{k=1}^{\infty}\left\{1-\lambda_{k} g\left(t_{k}\right) h\left(t_{k}\right)\right\}
$$

Proof. Since $f$ is non-decreasing and $g \geq I$ on $J,(2.1)$ may be written as

$$
\frac{x(t)}{f(t)} \leq g(t)\left[1+\int_{t_{0}}^{t} h(s) \frac{x(s)}{f(s)} d u(s)\right], \quad t \in J
$$

Denote the bracket on the right side of (2.5) by $r(t)$. Firstly suppose $t_{0} \leq t<t_{1}$. Since $u$ is differentiable on $\left[t_{0}, t_{1}\right]$, by the classical Gronwall-Bellman inequality [1, p. 58], we obtain

$$
r(t) \leq \exp \left(\int_{t_{0}}^{t} g(s) h(s) u_{1}^{\prime}(s) d s\right)
$$

At $t=t_{1}$ we have

$$
r\left(t_{I}\right)=r\left(t_{I}-\varepsilon\right)+\int_{t_{1}-\varepsilon}^{t_{I}} h(s) \frac{x(s)}{f(s)} d u(s),
$$

where $\varepsilon>0$. Taking the limit as $\varepsilon \rightarrow_{+}$and using (2.6), we get

$$
r\left(t_{1}\right) \leq \exp \left[\int_{t_{0}}^{t_{1}} g(s) h(s) u_{1}^{\prime}(s) d s\right\}+\lambda_{1} g\left(t_{1}\right) h\left(t_{1}\right) r\left(t_{1}\right) \text {, }
$$


which, in view of (2.2), yields

$$
r\left(t_{1}\right) \leq P_{1}^{-1} \exp \left(\int_{t_{0}}^{t_{1}} g(s) h(s) u_{1}^{\prime}(s) d s\right)
$$

where

$$
P_{k}=\prod_{n=1}^{k}\left\{1-\lambda_{n} g\left(t_{n}\right) h\left(t_{n}\right)\right\}, k=1,2, \ldots
$$

By mathematical induction, it follows that

$$
\text { (2.7) } \quad r\left(t_{m}\right) \leq P_{m}^{-1} \exp \left(\int_{t_{0}}^{t} g(s) h(s) u_{1}^{\prime}(s) d s\right), m=1,2, \ldots \text {. }
$$

Since $P_{i} \geq P_{i+1}$ for each $i \geq 1$ and $\lim _{i \rightarrow \infty} P_{i}=P$ (which exists in view of hypothesis (iii)), we may write (2.7) as

$$
r\left(t_{m}\right) \leq P^{-1} \exp \left(\int_{t_{0}}^{t_{m}} g(s) h(s) u_{1}^{\prime}(s) d s\right), m=1,2, \ldots .
$$

Now, given any $t \in J$, there is a unique integer $m \geq 0$ such that $t \in\left[t_{m}, t_{m+1}\right]$. Therefore

$$
\begin{aligned}
r(t) & =r\left(t_{m}\right)+\int_{\left.t_{m}, t\right]} h(s) \frac{x(s)}{f(s)} d u(s) \\
& \leq r\left(t_{m}\right) \exp \left\{\int_{t_{m}}^{t} g(s) h(s) u_{l}^{\prime}(s) d s\right\} .
\end{aligned}
$$

Hence we conclude that

$$
\begin{aligned}
x(t) & \leq f(t) g(t)_{r}(t) \\
& \leq P^{-1} f(t) g(t) \exp \left(\int_{t_{0}}^{t} g(s) h(s) u_{1}^{\prime}(s) d s\right), t \in J .
\end{aligned}
$$

This completes the proof.

As an illustration of Theorem 2.1, consider the inequality 


$$
x(t) \leq t^{2}+e^{t} \int_{1}^{t} 2 e^{-s}\left(s^{2}-s+1\right)^{-1} x(s) d u(s), \quad t \in[1, \infty)
$$

where

$$
u(t)=2^{-1}\left(t^{2}-t\right)+\left(\sum_{i=1}^{k-1}(i+1)^{-1}\right) x_{[k-1, k)}(t), \quad k=2,3, \ldots .
$$

Here $X_{A}$, the characteristic function of the set $A$, is defined as $X_{A}(t)=1$ if $t \in A$ and equal to zero otherwise. It is easily seen that $\mathcal{u}^{\prime}(t)=2^{-1}(2 t-1)$ almost everywhere on $[1, \infty) ; t_{k}=k, \lambda_{k}=(k+1)^{-1}$ for $k=2,3, \ldots ; \lambda_{k} g\left(t_{k}\right) h\left(t_{k}\right)=2\left(k^{3}+1\right)^{-1}<1$ for all $k \geq 2$; the series

$$
\sum_{k=2}^{\infty} 2\left(k^{3}+1\right)^{-1}<\infty
$$

by comparison test and

$$
P=\prod_{k=2}^{\infty}\left\{1-2\left(k^{3}+1\right)^{-1}\right\}=\frac{2}{3}
$$

Following the estimate in (2.4), we obtain

$$
x(t) \leq \frac{3}{2}\left(t^{4}-t^{3}+t^{2}\right) e^{t}, \text { for all } t \geq 1 \text {. }
$$

We apply Theorem 2.1, in the natural way, to Volterra integral equations of the form (1.1). To this end, we assume that there exist nonnegative functions $g$ and $h$ which are defined and locally du-integrable on $J$ and are such that

$$
\left|K\left(t, s, \phi_{1}\right)-K\left(t, s, \phi_{2}\right)\right| \leq g(t) h(s)\left|\phi_{1}-\phi_{2}\right|
$$

for all $\phi_{1}, \phi_{2} \in R^{n}$.

\section{THEOREM 2.2. Suppose that}

(i) (1.1) has a bounded solution $x$ defined on $J$,

(ii) $g \geq I$ is bounded on $J$ and 


$$
\int_{t_{0}}^{\infty} g(s) h(s) v_{1}^{\prime}(s) d s<\infty
$$

where $v=v_{1}+v_{2}$ is the decomposition of $v(t)=v\left(u(t),\left|t_{0}, t\right|\right)$, the total variation function of $u(t)$ on $\left|t_{0}, t\right|$,

(iii) $v_{1}^{\prime} \geq 0$ on $J$ and $\mu_{k} g\left(t_{k}\right) h\left(t_{k}\right)<1$, where $\mu_{k}=v\left(t_{k}\right)-v\left(t_{k}\right), k=1,2, \ldots$;

the series $\sum_{k=1}^{\infty} \mu_{k} g\left(t_{k}\right) h\left(t_{k}\right)$ converges absolutely.

If $f^{*} \in B V(J)$ is locally du-integrable on $J$ and $\left\|f(t)-f^{*}(t)\right\|$ is non-decreasing and bounded on $J$, then any solution of the equation

$$
y(t)=f^{*}(t)+\int_{t_{0}}^{t} K(t, s, y(s)) d u(s), \quad t \in J
$$

is bounded.

Proof. From (1.1), (2.8), and (2.10), we obtain

$$
\|x(t)-y(t)\| \leq\left\|f(t)-f^{*}(t)\right\|+g(t) \int_{t_{0}}^{t} h(s)\|x(s)-y(s)\| d v(s), \quad t \in J .
$$

Since $v$ is a right-continuous function of bounded variation and has discontinuities where $u$ has, a suitable application of Theorem 2.1 gives (2.11) $\|x(t)-y(t)\|$

$$
\leq P^{-1}\left\|f(t)-f^{*}(t)\right\| g(t) \exp \left(\int_{t_{0}}^{t} g(s) h(s) v_{1}^{\prime}(s) d s\right), \quad t \in J .
$$

As $x, g$, and $\left\|f-f^{*}\right\|$ are all bounded on $\mathcal{J}$, the conclusion follows from (2.9), (2.11), and the fact that $\|y(t)\| \leq\|y(t)-x(t)\|+\|x(t)\|$.

REMARK 2.1. A result similar to Theorem 2.2 is proved in [5, Theorem 3] where the integrals are in the Riemann-Stieltjes sense. Hence it is necessary that the integrand and the integrator should not have the same discontinuities. In our case, $x$ and $u$ have the same 
discontinuities and therefore the methods of [5] are not applicable.

3.

In this section, we consider a special case of (1.1), namely

$$
x(t)=f(t)+\int_{t_{0}}^{t} A(t, s) x(s) d u(s), \quad t \in J,
$$

where $A(t, s)$ is an $n \times n$ matrix defined for $t_{0} \leq s \leq t<\infty$. We show, under certain conditions, that (3.1) reduces to a difference equation. Choose $u$ to be a step function (that is $u_{1} \equiv 0$ ) of the form

$$
u(t)=\left(\sum_{i=0}^{k-1} a_{i}\right) x_{\left(t_{k-1}, t_{k}\right)}(t), k=1,2, \ldots,
$$

where the $a_{i}$ 's are constants. Let $J_{t_{0}}=\left\{t_{k}\right\}, k=0,1, \ldots$. Denote by $B_{k}$ the matrix $I-a_{k} A\left(t_{k}, t_{k}\right), k=1,2, \ldots$, where $I$ is the identity $n \times n$ matrix.

THEOREM 3.1. On $J_{t_{0}}$, (3.1) reduces to the difference equation

$$
\nabla x\left(t_{k}\right)=\nabla f\left(t_{k}\right)+a_{k} A\left(t_{k}, t_{k}\right) x\left(t_{k}\right), x\left(t_{0}\right)=f\left(t_{0}\right),
$$

where $\nabla$ is the operator such that $\nabla x\left(t_{k}\right)=x\left(t_{k}\right)-x\left(t_{k-1}\right)$. Furthermore, if $B_{k}$ is non-singular for each $k=1,2, \ldots$ then the unique solution of (3.2) is given by the recurrence formula

$$
x\left(t_{k}\right)=B_{k}^{-1}\left\{x\left(t_{k-1}\right)+\nabla f\left(t_{k}\right)\right\}, \quad k=1,2, \ldots .
$$

Proof. It is clear that $x\left(t_{0}\right)=f\left(t_{0}\right)$. For $t_{1} \in J_{t_{0}}$, we have from (3.1),

$$
\begin{aligned}
x\left(t_{1}\right) & =f\left(t_{1}\right)+\int_{t_{0}}^{t_{1}} A\left(t_{1}, s\right) x(s) d u(s) \\
& =f\left(t_{1}\right)+a_{1} A\left(t_{1}, t_{1}\right) x\left(t_{1}\right) .
\end{aligned}
$$

Similarly 


$$
\begin{aligned}
x\left(t_{2}\right) & =f\left(t_{2}\right)+\int_{t_{0}}^{t_{1}} A\left(t_{1}, s\right) x(s) d u(s)+\int_{t_{1}}^{t_{2}} A\left(t_{2}, s\right) x(s) d u(s) \\
& =x\left(t_{1}\right)+\nabla f\left(t_{2}\right)+a_{2} A\left(t_{2}, t_{2}\right) x\left(t_{2}\right) .
\end{aligned}
$$

In general, by induction,

(3.4) $x\left(t_{k}\right)=x\left(t_{k-1}\right)+\nabla f\left(t_{k}\right)+a_{k} A\left(t_{k}, t_{k}\right) x\left(t_{k}\right), k=1,2, \ldots$,

which is the same as (3.2). If $B_{k}$ is invertible, it follows from (3.4) that $x\left(t_{k}\right)$ exists uniquely and is given by (3.3).

REMARK 3.1. If, for some $k, a_{k}$ is zero, then $B_{k}(=I)$ is clearly invertible. If $A(t, s)=A$ is a constant matrix and if $a_{k} \neq 0$, then a sufficient condition for $B_{k}$ to be invertible is that $a_{k}^{-1}$ is not an eigenvalue of $A$.

REMARK 3.2. Suppose $B_{k}$ is not invertible for some $k$. Then it follows from (3.4) that, in general, $x\left(t_{k}\right)$ does not exist. On the other hand, if $x\left(t_{k-1}\right)+\nabla f\left(t_{k}\right)=0$, then $x\left(t_{k}\right)$ is arbitrarily determined, which means that there are infinitely many solutions at $t_{k}$. It is to be noted that if $f \equiv 0$, then $x\left(t_{k}\right)=0$ for each $k=0,1, \ldots$.

EXAMPLE 3.1. Let $f(t)=t$ and $A(t, s)=\left(e^{t}+t\right) \sin \frac{\pi s}{2}$. be scalar scalar functions on $[0, \infty)$. Choose

$$
u(t)=\left(\sum_{i=0}^{k-1} i^{-1}\right) x_{[k-1, k)}(t), k=1,2, \ldots .
$$

Then $u$ is discontinuous at isolated points $t_{k}=k$ and $a_{k}=k^{-1}$ for $k=1,2, \ldots$. The difference equation corresponding to (3.1) is

$$
\begin{aligned}
& x(0)=0, \\
& x(k)=x(k-1)+1+k^{-1}\left(e^{k}+k\right) \sin \frac{k \pi}{2} x(k), k=1,2, \ldots .
\end{aligned}
$$

Since $\left(e^{k}+k\right) \sin \frac{k \pi}{2} \neq k$ for any $k \geq 1$, the condition of Theorem 3.1 is satisfied. $x(k)$ can now be determined from (3.3). 
EXAMPLE 3.2. Let $A(t, s)=A$ be the constant matrix

$$
\left[\begin{array}{cc}
2 & -1 / 3 \\
-6 & 1
\end{array}\right]
$$

and

$$
u(t)=\frac{2}{3} x_{[0,1)}(t)+\left\{\sum_{i=1}^{k-1} i^{2}\right\} x_{[k-1, k)}(t), k=2,3, \ldots .
$$

Here $a_{1}^{-1}=3$ is an eigenvalue of $A$, the corresponding eigenvector being $\left[\begin{array}{c}1 \\ -3\end{array}\right]$. If $f(1) \neq\left[\begin{array}{l}0 \\ 0\end{array}\right], x(1)$ does not exist. Moreover, $c\left[\begin{array}{c}1 \\ -3\end{array}\right]$ is also an eigenvector where $c$ is any constant. Therefore, if $f(1)=\left[\begin{array}{l}0 \\ 0\end{array}\right]$ then $x(1)=c\left[\begin{array}{c}1 \\ -3\end{array}\right]$, meaning thereby that there are infinitely many solutions.

4.

In this section, we obtain sufficient conditions in order that a certain stability of the system (1.2) implies the corresponding local stability of the system (1.3). The solutions $y(t)$ and $x(t)$ of (1.2) and (1.3) are respectively given by (the variation of constants formula)

$$
y(t)=f(t)+\int_{t_{0}}^{t} R(t, s) f(s) d s, t \geq t_{0},
$$

and

(4.2) $\quad x(t)=y(t)+\int_{t_{0}}^{t} R^{*}(t, s) F(s, x(s)) d u(s), t \geq t_{0}$,

where $R(t, s)$ and $R^{*}(t, s)$ satisfy

$$
R(t, s)=a(t, s)+\int_{s}^{t} R(t, \tau) a(\tau, s) d \tau
$$

and 
$(4.4)$

$$
R^{*}(t, s)=b(t, s)+\int_{s}^{t} R^{*}(t, \tau) b(\tau, s) d \tau .
$$

The main result (Theorem 4.1) of this section depends on the following lemma, which is interesting in itself.

\section{LEMMA 4.1. Assume}

(i) $x, f$, and $u$ are as in Theorem 2.1,

(ii) $g_{i}, h_{i}$ are non-negative functions, locally du-integrable on $J$, and $g_{i} \geq 1$ for $i=1,2, \ldots, n$,

(iii) $\lambda_{k} g_{i}\left(t_{k}\right) h_{i}\left(t_{k}\right)<1$ for $k \geq 1$ and the $n$ series

$$
\sum_{k=1}^{\infty} \lambda_{k} g_{i}\left(t_{k}\right) h_{i}\left(t_{k}\right)
$$

converge absolutely for $i=1,2, \ldots, n$.

Then the inequality

(4.5) $\quad x(t) \leq f(t)+\sum_{i=1}^{n} g_{i}(t) \int_{t_{0}}^{t} h_{i}(s) x(s) d u(s), \quad t \in J$,

implies

$$
x(t) \leq P^{-1} E_{f}^{n}
$$

where

$$
\begin{aligned}
E^{0} f & =f, \\
E^{r} f & =f\left(E^{r-1} g_{r}\right) \exp \left(\int_{t_{0}}^{t} h_{r}\left(E^{r-1} g_{r}\right) u_{i}^{\prime}(s) d s\right\}, r=1,2, \ldots, n, \\
P_{i} & =\prod_{k=1}^{\infty}\left\{1-\lambda_{k} g_{i}\left(t_{k}\right) h_{i}\left(t_{k}\right)\right\}, i=1,2, \ldots, n,
\end{aligned}
$$

and

$$
P=P_{1} P_{2} \cdots P_{n}
$$

The proof can be obtained by applying Theorem 2.1 and the method of Theorem $I$ in [4]. We omit the details. 
Now consider equations (1.2) and (1.3) whose zolutions are given by (4.1) and (4.2) respectively. Assume that

$\mathrm{H}_{1}$. There exists $r>0$ such that

$$
|F(t, x)| \leq f(t)\|x\| \text { for } t \geq t_{0} \text { and }\|x\|<r \text {, }
$$

where $f(t)$ is non-negative and du-integrable on $J$.

$\mathrm{H}_{2}$. $R^{*}$ satisfies

$$
\left|R^{*}(t, s)\right| \leq \sum_{i=1}^{n} g_{i}(t) h_{i}(s) \text { for } t_{0} \leq s \leq t<\infty,
$$

where, for $i=1,2, \ldots, n, g_{i}, h_{i}$ are non-negative functions, du-integrable on $J$, and $g_{i} \geq 1 ; \mu_{k} f\left(t_{k}\right) g_{i}\left(t_{k}\right) h_{i}\left(t_{k}\right)<1$ for $k \geq 1$, and the $n$ series

$$
\sum_{k=1}^{\infty} \mu_{k} f\left(t_{k}\right) g_{i}\left(t_{k}\right) h_{i}\left(t_{k}\right)
$$

converge absolutely where $\mu_{k}$ is as defined in Theorem 2.2.

THEOREM 4.1. Under the hypotheses $\mathrm{H}_{1}$ and $\mathrm{H}_{2}$, any solution $x$ of (1.3) satisfies

$$
\|x(t)\| \leq P^{-1} E^{n}\|y\|
$$

where $y$ is any solution of (1.2); $E^{n}$ is as defined in Lemma 4.1 except that $u_{1}^{\prime}$ is replaced by $v_{1}^{\prime}$;

$P_{i}=\prod_{k=1}^{\infty}\left\{1-\mu_{k} f\left(t_{k}\right) g_{i}\left(t_{k}\right) h_{i}\left(t_{k}\right)\right\}, i=1,2, \ldots, n$ and $P=P_{1} P_{2} \ldots P_{n}$.

Proof. We have

$$
\|x(t)\| \leq\|y(t)\|+\sum_{i=1}^{n} f(t) g_{i}(t) \int_{t_{0}}^{t} h_{i}(s)\|x(s)\| d v(s), t \in J .
$$

Since $\|y(t)\|$ is non-decreasing on $J$, an application of Lemma 4.1 gives the desired conclusion.

REMARK 4.1. Theorem 4.1 may be regarded as a result on local 
stability of the system (1.3) with respect to the system (1.2) in the following sense: given $\delta>0$ and sufficiently small, the solution $x$ of (1.3) satisfies $\|x(t)\|<c \delta, c>0, t \geq t_{0}$, whenever $\|y(t)\|<\delta$.

As an illustration of Lemma 4.1, consider the inequality

$$
x(t) \leq e^{t}+t \int_{1}^{t} s^{-2} x(s) d u(s)+t^{2} \int_{1}^{t}\left(4 s^{3}\right)^{-1} x(s) d u(s),
$$

where

$$
u(t)=t+\left\{\sum_{i=1}^{k-1} i^{-1}\right\} x_{[k-1, k)}(t), \quad k=2,3, \ldots .
$$

Here $t_{k}=k, \lambda_{k}=k^{-1}$ for $k=2,3, \ldots$;

$$
\begin{aligned}
& P_{1}=\prod_{k=2}^{\infty}\left(1-k^{-2}\right)=1 / 2 ; \\
& P_{2}=\prod_{k=2}^{\infty}\left(1-\frac{1}{4 k^{2}}\right)=\frac{8}{3 \pi} .
\end{aligned}
$$

In view of $(4.6)$, we obtain

$$
x(t) \leq P^{-1} E^{2} f=\frac{3 \pi}{4} t^{4} \exp \left(\frac{t^{2}+8 t-1}{8}\right) \text {, for all } t \geq 1 .
$$

REMARK 4.1. Lemma 4.1 has a distinct advantage over Theorem 2.1. To see this, consider the inequality (4.8). Since $t \geq s \geq 1$, we may write it as

$$
x(t) \leq e^{t}+2 t^{2} \int_{1}^{t} s^{-2} x(s) d u(s), \quad t \geq 1,
$$

which is of the form (2.1). In the notation of Theorem 2.1, we see that $\lambda_{k} g\left(t_{k}\right) h\left(t_{k}\right)=2 k^{-1}, k=2,3, \ldots$. However, Theorem 2.1 is not applicable here for two reasons; firstly because $\lambda_{2} g\left(t_{2}\right) h\left(t_{2}\right) \nless 1$, and secondly because the series $\sum_{k=2}^{\infty} 2 k^{-1}$ diverges. 


\section{References}

[1] Richard E. Bell Iman and Kenneth L. Cooke, Differential-difference equations (Academic Press, New York, London, 1963).

[2] Constantin Corduneanu, Integral equations and stability of feedback systems (Mathematics in Science and Engineering, 104. Academic Press [Harcourt Brace Jovanovich], New York and London, 1973).

[3] P.C. Das and R.R. Sharma, "Existence and stability of measure differential equations", Czechoslovak Math. J. 22 (97) (1972), $145-158$.

[4] U.D. Dhongade and S.G. Deo, "Pointwise estimates of solutions of some Volterra integral equations", J. Math. Anal. Appl. 45 (1974), $615-628$.

[5] G. Stephen Jones, "Fundamental inequalities for discrete and discontinuous functional equations", J. Soc. Indust. App Z. Math. 12 (1964), 43-57.

[6] R.K. Miller, J.A. Nohel, and J.S.W. Wong, "Perturbations of Volterra integral equations", J. Math. AnaZ. App Z. 25 (1969), 676-691.

[7] S.G. Pandit, "On the stability of impulsively perturbed differential systems", BulZ. Austral. Math. Soc. 17 (1977), $423-432$.

[8] V. Raghavendra and M. Rama Mohana Rao, "Integral equations of Volterra type and admissibility theory", Rev. Rowm. Math. Pure App 2. 18 (1973), 571-580.

[9] V. Raghavendra and M. Rama Mohana Rao, "Volterra integral equations with discontinuous perturbations", Mathematica (Cluj) (to appear).

[10] Aaron Strauss, "On a perturbed Volterra integral equation", J. Math. Ana Z. Appl. 30 (1970), 564-575.

Department of Mathematics,

Centre of Post-Graduate Instruction and Research,

University of Bombay,

Panaji, Goa,

India. 